\title{
An Atypical Presentation of Dermatomyositis Sine Der- matitis in A Child with Autism
}

\author{
Laurel G Wolf*, Ariel Breitbart, Mileka Gilbert, Patricia McBurney \\ Department of Pediatrics, Medical University of South Carolina, USA
}

*Corresponding author: Laurel G Wolf, Department of Pediatrics, Medical University of South Carolina, USA.

Received Date: May 17, 2021

Published Date: August 23, 2021

\begin{abstract}
Background: Juvenile Dermatomyositis (JDM) is a rare inflammatory myopathy that classically presents with proximal muscle weakness and classic rashes, however there is a form without rash, "sine dermatitis." There are also relatively recently described myositis-specific autoantibodies, such as NXP-2 antibody, that can be present in JDM. Variations in clinical presentation and associations of this myositis-specific autoantibody have not been extensively described.
\end{abstract}

Case Presentation: A 5-year-old female with a history of autism spectrum disorder (ASD) and recent travel to Jamaica presented with 2.5 months of left lower extremity weakness and more recent onset of left upper extremity weakness. Her physical exam was significant for left upper and lower extremity pain and decreased range of motion, absence of skin rash, as well as communication deficits secondary to ASD. She underwent an extensive workup including evaluation for infectious, neurologic, and rheumatologic etiologies of her weakness. Initial laboratory evaluation revealed elevated inflammatory markers, mildly elevated creatine kinase, and positive antinuclear antibody. Magnetic resonance imaging showed evidence of bilateral diffuse myositis with subsequent muscle biopsy of left lower extremity showing signs of an inflammatory myopathy. Further laboratory evaluation was positive for NXP-2 autoantibody associated with JDM. Her clinical picture is most consistent with JDM sine dermatitis. She was treated with steroid taper and methotrexate with improvement in symptoms.

Conclusion: JDM can present in various ways, and clinical suspicion should be high even in patients with misleading historical and physical exam findings. Because JDM is such a rare disease, this case provides important insight into the atypical way that it can present, particularly with a challenging physical exam, and it provides information on clinical manifestations appreciated with the NXP-2 autoantibody.

Keywords: Juvenile dermatomyositis; Dermatomyositis sine dermatitis; Juvenile idiopathic inflammatory myopathy; NXP-2 antibody. Abbreviations: ASD Autism Spectrum Disorder; ANA Antinuclear Antibody; CK Creatine Kinase; ESR Erythrocyte Sedimentation Rate; LDH Lactate Dehydrogenase; JIIM Juvenile Idiopathic Inflammatory Myopathy; JDM Juvenile Dermatomyositis; JPM Juvenile Polymyositis; AST Aspartate Aminotransferase; ALT Alanine Aminotransferase; EULAR European League Against Rheumatism; ACR American College of Rheumatology

\section{Introduction}

Juvenile idiopathic inflammatory myopathies (JIIMs) are rare childhood chronic autoimmune rheumatic diseases. Juvenile dermatomyositis (JDM) is the most common of the JIIMs with an incidence of 3.2 per million children per year. Other subtypes of IIMs include focal myositis, polymyositis, inclusion body myositis, immune-mediated necrotizing myopathy, anti-synthetase syndrome, and overlap syndromes [1-3].

JDM is clinically characterized by symmetric proximal muscle weakness, classic rashes (including malar, heliotrope,

Gottron's sign, and Gottron's papules), and nailfold capillaropathy, although disease can include musculoskeletal, pulmonary, and gastrointestinal manifestations. However, JDM can have variable presentations that include an amyopathic form or sine dermatitis. Laboratory findings consistent with muscle breakdown include elevations in serum creatine kinase (CK), lactate dehydrogenase (LDH), aspartate aminotransferase (AST), alanine aminotransferase (ALT), and aldolase. EULAR/ACR criteria have been used to diagnose inflammatory myopathy, however these criteria only denote risk of disease and do not rule out disease if negative. More 
recently, presence of myositis-specific autoantibodies has been used to attempt to distinguish phenotypes of JIIMs. In particular, NXP-2 antibody is a novel autoantibody that has previously been associated with calcinosis, joint contractures, and polyarthritis in JIIM [1-3]. In this report, we describe a patient with an atypical presentation of JDM sine dermatitis and the presence of the novel myositis specific autoantibody NXP-2 without the previously described associations.

\section{Case Presentation}

A 5-year-old female with a past medical history of autism spectrum disorder (ASD) and recent travel history to Jamaica presented with limp and left-sided weakness for 2.5 months. The patient's symptoms started with limp when she was in Jamaica visiting family and progressed to weakness, stiffness, refusal to bear weight on the left lower extremity, then requiring use of a wall to assist with standing from a seated position. She also had more recent decreased use of her left upper extremity and kept her arm flexed at the elbow. She had no associated joint swelling, erythema, tenderness, rash, or fever. She was up-to-date on vaccinations. When she returned from Jamaica, she was evaluated by an orthopedic surgeon in clinic who recommended physical therapy, but she had no clinical improvement. Her primary care doctor obtained lab work which was significant for positive antinuclear antibody (ANA) 1:2560 titer (speckled pattern), CK $238 \mathrm{U} / \mathrm{L}$, and erythrocyte sedimentation rate (ESR) $82 \mathrm{~mm} /$ hour, prompting presentation to the Emergency Department.

On admission, she was afebrile and hemodynamically stable. Physical exam was extremely limited by her poor communication secondary to ASD; however, pain and decreased range of motion in the left lower and upper extremities were observed. Physical exam was also significant for toe walking, which was present at baseline due to her ASD, and a left-sided antalgic gait. Radiograph of left knee and elbow were unrevealing. The differential diagnosis at this time was quite broad and included rheumatologic, infectious, neurologic, metabolic (heavy metals, vitamin deficiency), and malignant causes.

Rheumatology was consulted and recommended laboratory evaluation to look for autoimmune processes as a potential cause of her symptoms. These were significant for elevated CK (239 U/L), elevated LDH (467 U/L), elevated aldolase (10.8 U/L), slightly elevated complement levels (C3 $154.7 \mathrm{mg} / \mathrm{dL}, \mathrm{C} 443.4 \mathrm{mg} / \mathrm{dL}$ ), hypoalbuminemia ( $3.2 \mathrm{~g} / \mathrm{dL}$ ), and normocytic anemia (hemoglobin 10.5 g/dL). Jo-1, Sm, RNP, dsDNA, SSA, and SSB antibodies were all negative. Serum transaminases and urinalysis were normal. MRI with and without contrast of the left lower extremity was obtained to evaluate for osteomyelitis, arthritis, and myositis and showed extensive hyperintensity and enhancement in gluteal, hip abductors, and upper leg musculature representing myositis or myopathy. Included portions of the right lower extremity showed the same signal abnormalities.
With this new information and due to her recent travel history, infectious workup, including testing for Echovirus, Coxsackie virus, human immunodeficiency virus, human t-cell lymphotropic virus type 1, Dengue virus, Zika virus, and Chikungunya virus, was performed, and tests were all negative. Simultaneously, a muscle biopsy of the left thigh was pursued and significant for interstitial perivascular mononuclear inflammatory infiltrates consistent with an inflammatory myopathy. There were no myotubes, myophagocytosis, perifascicular atrophy, cytoplasmic inclusions by trichrome stain, or target fibers. The DPAS and PAS stains showed a normal glycogen content. Neuropathic changes such as group atrophy were not found. Electromyography was not pursued because of patient's difficulty cooperating with exam due to ASD. She was started on scheduled naproxen $10 \mathrm{mg} / \mathrm{kg}$ twice daily and daily oral prednisolone $0.6 \mathrm{mg} / \mathrm{kg}$ daily due to concern for JIIM. Pain and range of motion improved, and the patient was able to discharge home with rheumatology follow up.

After discharge, myositis-specific autoantibody panel resulted positive for NXP-2 antibody, which is associated with juvenile polymyositis (JPM) and JDM. Given that the muscle biopsy pathology was more consistent with DM, and that the patient never had a rash (malar, heliotrope, or Gottron's rash) throughout her disease course, her clinical picture is most consistent with JDM sine dermatitis. She has also been treated with injectable methotrexate $\sim 15 \mathrm{mg} / \mathrm{m} 2$ weekly as an immunosuppressant agent along with physical therapy. These have led to improvement in symptoms, serum muscle enzymes, and allowed for prednisolone taper.

\section{Discussion}

JDM classically presents with signs and symptoms of proximal muscle weakness based on history and physical exam. This patient's presentation was unique because her physical exam and laboratory findings were not typical for inflammatory myopathy. In particular, her muscle weakness appeared to be unilateral instead of the typical symmetric pattern that is seen in JDM, and she seemed to have pain with passive and active range of motion. It is possible that the interpretation of her exam was altered by behavioral concerns with her history of ASD. Additionally, she did not have any skin findings that would point to the much more common classic presentation of JDM. Her labs did show slightly elevated CK, elevated aldolase, and elevated LDH, but she did not have elevated AST/ALT and CK elevations tend to be much higher in polymyositis. Jo-1 antibody, associated with DM, was negative, however this is rare in childhood-onset dermatomyositis.

In 2017, the European League Against Rheumatism (EULAR) and the American College of Rheumatology (ACR) published classification criteria for adult and juvenile idiopathic inflammatory myopathy [4]. The criteria include physical exam findings, such as muscle weakness and skin manifestations, as well as laboratory measurements and other clinical manifestations like dysphagia (Table 1). Because some providers are pursuing muscle biopsy less 
frequently than in the past, the EULAR/ACR criteria can be scored with or without a muscle biopsy.

Utilizing the EULAR/ACR criteria for this patient (Table 1), her objective findings do not suggest increased risk of idiopathic inflammatory myopathy either with or without using the results of her muscle biopsy. Even though her muscle biopsy was consistent with an idiopathic inflammatory myopathy - showing interstitial perivascular mononuclear inflammatory infiltrates - her apparent unilateral physical exam findings and lack of skin manifestations kept her score from being above the cutoff for increased risk of idiopathic inflammatory myopathy. It is unclear how communication and behavior could have played a role in the objective exam for assessing symmetric muscle weakness, and it is important to note that the MRI did show symmetric proximal muscle involvement consistent with JIIM.

Table 1: EULAR/ACR Criteria for this Specific Patient Prior to muscle biopsy her score was 2.2, which correlates with a $<50 \%$ chance of having the disease. A score of $\geq 5.5$ would correlate with increased risk of having inflammatory myopathy. After muscle biopsy, her score was 3.8 , which similarly denotes a low chance of having the disease. A score of $\geq 6.7$ is associated with increased risk of disease. (CK creatine kinase, LDH lactate dehydrogenase, AST aspartate aminotransferase, ALT alanine aminotransferase).

Variable Score Points without muscle biopsy $\quad$ Score points with muscle biopsy

\begin{tabular}{|c|c|c|}
\hline \multicolumn{3}{|c|}{ Age of onset } \\
\hline Age of onset of first symptom between 18 and 40 years old & 0 & 0 \\
\hline Age of onset $>/=40$ years old & 0 & 0 \\
\hline \multicolumn{3}{|c|}{ Muscle weakness } \\
\hline $\begin{array}{l}\text { Objective symmetric weakness, usually progressive of the proximal } \\
\text { upper extremities }\end{array}$ & 0 & 0 \\
\hline $\begin{array}{c}\text { Objective symmetric weakness, usually progressive, of the proximal } \\
\text { lower extremities }\end{array}$ & 0 & 0 \\
\hline Neck flexors are relatively weaker than neck extensors & 0 & 0 \\
\hline $\begin{array}{l}\text { In the legs, proximal muscles are relatively weaker than distal mus- } \\
\text { cles }\end{array}$ & 0.9 & 1.2 \\
\hline \multicolumn{3}{|c|}{ Skin Manifestations } \\
\hline Heliotrope rash & 0 & 0 \\
\hline Gottron's papules & 0 & 0 \\
\hline Gottron's sign & 0 & 0 \\
\hline \multicolumn{3}{|c|}{ Other clinical manifestations } \\
\hline Dysphagia or esophageal dysmotility & 0 & 0 \\
\hline \multicolumn{3}{|c|}{ Laboratory Measurements } \\
\hline Anti Jo 1 autoantibody present & 0 & 0 \\
\hline Elevated levels of CK or LDH or AST/ALT & 1.3 & 1.4 \\
\hline \multicolumn{3}{|c|}{ Muscle biopsy features - presence of: } \\
\hline $\begin{array}{l}\text { Endomysial infiltration of mononuclear cells surrounding, but not } \\
\text { invading, myofibres }\end{array}$ & & 0 \\
\hline Perimysial and/or perivascular infiltration of mononuclear cells & & 1.2 \\
\hline Perifascicular atrophy & & 0 \\
\hline Rimmed vacuoles & & 0 \\
\hline TOTAL & 2.2 & 3.8 \\
\hline
\end{tabular}

The positive NXP-2 antibody and lack of rash in the setting of abnormal MRI and muscle biopsy helped to confirm the diagnosis of JDM sine dermatitis in this patient, even though EULAR/ACR criteria denoted low risk of disease. NXP-2 antibody is specific for idiopathic inflammatory myopathies and is diagnostic in the right clinical scenario. NXP-2 antibody is a relatively recently described myositis-specific autoantibody and is not currently included in the EULAR/ACR criteria [5]. The antibody was first described in a cohort of patients with JDM in 1997 [6] and was associated with severe calcinosis, polyarthritis, joint contractures, and intestinal vasculitis.
Since then, NXP-2 antibody has been documented in various other cohorts in both pediatric and adult patients [5]. Because of the relative novelty of this antibody, its disease associations have not been well described. Positive NXP-2 antibodies were more commonly identified in JDM in case series but were also seen in few patients with JPM $[7,8]$. Additionally, $86 \%$ of patients with DM sine dermatitis were positive for NXP-2 antibody in a small cohort of 14 patients [9]. Patients with NXP-2 antibodies had more severe disease at follow up, including severe atrophy with contractures, large joint arthritis, and dysphagia, although the study did not 
distinguish these characteristics between patients with JDM and JPM [10]. The patient presented in this report did not have any calcinosis, severe atrophy, or other stigmata of severe disease, however she did have decreased range of motion in her upper and lower extremities which denotes contractures. This supports the notions that these manifestations take time to develop, there is a broad spectrum of associated disease manifestations, and there is still much unknown about this particular autoantibody.

There were multiple confounding factors in this case that made this patient's diagnosis a difficult one. The most significant factor was her diagnosis of ASD that limited the physical exam and the patient's ability to communicate about her symptoms. Her primary symptom of weakness was difficult to parse out and could have easily been mistaken for solely pain. Additionally, she was assessed as having unilateral symptoms, however bilateral MRI findings would suggest that symptoms were in fact bilateral and limited by communication and participation in exam. During her workup, there was significant thought given to the idea of a genetic disorder that may have been misdiagnosed as ASD. On further research, the patient had received formal neurodevelopmental testing to confirm ASD and had been tested for appropriate genetic causes of ASD. Although there are no case reports of ASD and JDM, there is much research into the role of the immune system and inflammation in ASD [11].

Another confounding factor to her presentation was her recent international travel history. The patient's symptoms started while she was visiting family in Jamaica, therefore there was concern for an infectious etiology. Her infectious workup was negative; however, the possibility remains that there was some infectious agent that prompted the onset of her myositis.

In conclusion, this patient presented with atypical signs and symptoms of JIIM including unilateral weakness, pain, and no dermatologic signs. Based on EULAR/ACR criteria, she was at low risk for JIIM. Her clinical picture was confounded by her recent travel history and her perceived unilateral muscle weakness likely affected by her diagnosis of ASD. Ultimately, diagnosis was confirmed as JDM sine dermatitis based on MRI, muscle biopsy, and serum antibody testing. This relatively novel autoantibody has not been well-described, and this case report offers insight into an atypical presentation of JDM associated with positive NXP-2 antibody.

\section{Acknowledgements}

Dr. Wolf collected data, conceptualized, and designed the case report, drafted the initial manuscript, and reviewed and revised the manuscript. Drs. Breitbart, Gilbert, and McBurney substantially contributed to the analysis and interpretation of data and reviewed and revised the manuscript. All authors approved the final manuscript as submitted and agree to be accountable for all aspects of the work.

\section{Conflict of Interest}

The authors do not have any financial interest or conflicts of interest.

\section{References}

1. Huber A, Feldman BM (2013) An update on inflammatory myositis in children. Curr Opin Rheumatol 25: 630-635.

2. Feldman BM, Rider LG, Reed AM, Pachman LM (2008) Juvenile dermatomyositis and other idiopathic inflammatory myopathies of childhood. Lancet 371(9631): 2201-2212.

3. Tanboon J, Nishino I (2019) Classification of idiopathic inflammatory myopathies: pathology perspectives. Curr Opin Neurol 32(5): 704-714.

4. Lundberg IE, Tjarnlund A, Bottai M, Victoria P Werth, Clarissa Pilkington et al. (2017) European League Against Rheumatism/American College of Rheumatology classification criteria for adult and juvenile idiopathic inflammatory myopathies and their major subgroups [published correction appears in Ann Rheum Dis. 2018 Sep;77(9): e64]. Ann Rheum Dis 76(12): 1955-1964

5. Satoh M, Tanaka S, Ceribelli A, Calise SJ, Chan EK (2017) A Comprehensive Overview on Myositis-Specific Antibodies: New and Old Biomarkers in Idiopathic Inflammatory Myopathy. Clin Rev Allergy Immunol 52(1): 1-19.

6. Oddis CV, Fertig N, Goel A, Espada G, Confalone Gregorian M, et al. (1997) Clinical and serological characterization of the anti-MJ antibody in childhood myositis. Poster presented at: American College of Rheumatology Conference Washington, DC.

7. Gunawardena H, Wedderburn LR, Chinoy H, Z. E. Betteridge, J. North, et al. (2009) Autoantibodies to a 140-kd protein in juvenile dermatomyositis are associated with calcinosis. Arthritis Rheum 60(6): 1807-1814.

8. Espada G, Maldonado Cocco JA, Fertig N, Oddis CV (2009) Clinical and serologic characterization of an Argentine pediatric myositis cohort: identification of a novel autoantibody (anti-MJ) to a 142 -kDa protein. J Rheumatol 36(11): 2547-2551.

9. Inoue M, Tanboon J, Hirakawa S, Hirofumi Komaki, Takeshi Fukushima, et al. (2020) Association of Dermatomyositis Sine Dermatitis With AntiNuclear Matrix Protein 2 Autoantibodies. JAMA Neurol 77(7): 872-877.

10. Espada G, Maldonado Cocco JA, Fertig N, Oddis CV (2009) Clinical and serologic characterization of an Argentine pediatric myositis cohort: identification of a novel autoantibody (anti-MJ) to a $142-\mathrm{kDa}$ protein. J Rheumatol 36(11): 2547-2551.

11. Meltzer A, Van de Water J (2017) The Role of the Immune System in Autism Spectrum Disorder. Neuropsychopharmacology: official publication of the American College of Neuropsychopharmacology 42(1): 284-298. 